\title{
Three-dimensional modeling of acoustic backscattering from fluid-like zooplankton
}

\author{
Andone C. Lavery, Timothy K. Stanton, Duncan E. McGehee, ${ }^{\text {a) }}$ and Dezhang Chu \\ Woods Hole Oceanographic Institution, Woods Hole, Massachusetts 02543
}

(Received 23 March 2001; revised 19 October 2001; accepted 13 November 2001)

\begin{abstract}
Scattering models that correctly incorporate organism size and shape are a critical component for the remote detection and classification of many marine organisms. In this work, an acoustic scattering model has been developed for fluid-like zooplankton that is based on the distorted wave Born approximation (DWBA) and that makes use of high-resolution three-dimensional measurements of the animal's outer boundary shape. High-resolution computerized tomography (CT) was used to determine the three-dimensional digitizations of animal shape. This study focuses on developing the methodology for incorporating high-resolution CT scans into a scattering model that is generally valid for any body with fluid-like material properties. The model predictions are compared to controlled laboratory measurements of the acoustic backscattering from live individual decapod shrimp. The frequency range used was $50 \mathrm{kHz}$ to $1 \mathrm{MHz}$ and the angular characteristics of the backscattering were investigated with up to a $1^{\circ}$ angular resolution. The practical conditions under which it is necessary to make use of high-resolution digitizations of shape are assessed. (C) 2002 Acoustical Society of America. [DOI: 10.1121/1.1433813]
\end{abstract}

PACS numbers: 43.30.Ft, 43.30.Sf, 43.20.Fn [DLB]

\section{INTRODUCTION}

Acoustic surveys are commonly used as a tool for assessing the distribution of zooplankton and other marine organisms that inhabit the water column. The primary advantage of this technique over other more conventional methods, such as net tows, is the possibility of performing synoptic surveys while also obtaining high-resolution data over relevant temporal and spatial scales (Medwin and Clay, 1998; Foote and Stanton, 2000). To accurately measure zooplankton biomass using this technique, it is necessary to first understand the process by which zooplankton scatter sound. Understanding the scattering of sound from zooplankton is a challenge for a number of reasons. First, many different species of zooplankton are typically present in the water column at any given time and location. Second, the scattering properties of these different species can vary enormously with organism size, shape, orientation, material properties, and acoustic frequency. Under many circumstances, successful interpretation of acoustic field data is limited in part by the availability of acoustic scattering models that take into account these diverse scattering properties. Though it is not feasible to develop a scattering model for each individual animal, it is possible to establish a categorization scheme based on general scattering properties and boundary conditions. One such scheme was developed by Stanton et al. (1994, 1998a, 1998b) in which three categories were identified; zooplankton with fluid-like acoustic characteristics (e.g., euphausiids, copepods), zooplankton with gas inclusions (e.g., siphonophores), and zooplankton with elastic shells (e.g., pteropods).

The investigation focuses on organisms with fluid-like material properties, meaning that shear waves are not in-

\footnotetext{
${ }^{a)}$ Currently at BAE Systems, Analysis \& Applied Research Division, San Diego, CA 92123.
}

cluded in the formalism. It is also assumed that there are no gas inclusions. Although the shape of the animals investigated in this paper is elongated, the model is not restricted to elongated scatterers, but is generally applicable to any fluidlike body, of any complex shape and size. Elongated fluidlike zooplankton are a possible application of this general model and are of particular interest due to the ecological significance and naturally high abundances found in many regions (Greene et al., 1991; Hewitt and Demer, 1991). Specifically, in this study we will present data and modeling results obtained for common shore shrimp, Palaeomonetes vulgaris. This crustacean has fluid-like material properties and a similar shape and size to many elongated fluid-like zooplankton, and has the added advantage of being conveniently obtainable from local waters. In addition, previous models based on this local species have been adapted with some success to oceanic species. There have been a number of laboratory and field studies aimed at understanding acoustic scattering from fluid-like scatterers, concentrating predominantly on decapod shrimp and euphausiids (Greenlaw, 1977; Everson, 1982; Everson et al., 1990; Foote et al., 1990; Chu et al., 1992, 1993; Martin Traykovski et al., 1998; McGehee et al., 1998; Stanton et al., 1993a, 1993b, 1996, 1998b, 1998c, 2000).

In a recent paper, Stanton and Chu (2000) presented a comprehensive review of the scattering models currently available for predicting the scattering of sound from elongated fluid-like zooplankton. Briefly synthesizing this work, the Anderson (1950) homogeneous fluid-sphere model is the simplest possible representation for animal shape used to describe scattering from this class of zooplankton (Greenlaw, 1977; Holliday et al., 1989; Holliday and Pieper, 1995). In a series of studies, cited in Stanton and Chu (2000), Stanton and others have developed a number of models that make use of finite-length deformed cylinders to describe animal shape, resulting in a more accurate description of the ob- 
served angular scattering characteristics. To date, the most sophisticated model available for this class of zooplankton, with the largest range of applicability, is a deformed-cylinder model based on the distorted wave Born approximation (DWBA) (Chu et al., 1993; Stanton et al., 1993b, 1998a, 1998b; McGehee et al., 1998; Chu and Ye, 1999; Stanton and $\mathrm{Chu}, 2000$ ). With this model it is possible to incorporate a relatively realistic animal shape, in addition to variations in the material properties, though a fundamental restriction of this model is the assumption of cylindrical symmetry. A result of this limitation is that the animal cross section is circular at every point along the lengthwise axis of the animal, and the material properties can only vary along the lengthwise axis.

In this study, we develop a DWBA-based scattering model that incorporates high-resolution, fully threedimensional (3D) digitizations of animal shape obtained through the use of computerized tomography (CT). This model by-passes the limitations imposed by the assumption of cylindrical symmetry made in the finite-length deformedcylinder model. We also compare the model predictions to acoustic backscattering laboratory measurements of both individual and aggregations of live decapod shrimp. The data presented in this paper were obtained during two separate experiments. The most recent data set, described in detail and for the first time in this paper, was obtained for live individuals under well-characterized and controlled conditions, using both single-frequency and broadband signals, with the animal orientation carefully controlled and with an angular resolution of $1^{\circ}$ in two scattering planes. The second data set used in this paper to compare with the model predictions was previously published by Stanton et al. (1993b) and involves aggregations of live decapod shrimp. Though the acoustics and CT data were obtained on animals of the same species and of generally the same shape and size, for practical reasons the acoustics and CT data involved different specimens.

This paper is organized as follows. In Sec. II, two DWBA-based scattering models are described. One of these models makes use of 3D measurements of the animal shape and results in a volume-integral solution; the other model, the deformed-cylinder model, uses 2D measurements of animal shape and results in a line-integral solution. In Sec. III, the laboratory setup, methods used for data collection and analysis, and the methods used to obtain 2D and 3D measurements of animal shape are outlined. Intermodel comparisons are performed in Sec. IV. Scattering predictions are compared to the data in Sec. V, and the physics of the scattering process is discussed in Sec. VI. Finally, in Sec. VII, the results are summarized, and recommendations are made regarding conditions under which the various approaches should be used.

\section{THEORY}

\section{A. DWBA-based scattering model}

For a single insonification of a target of any complex shape and size, the incident and scattered sound wave are related by the scattering amplitude. The scattering amplitude, which has units of length, is a measure of the efficiency with which a target scatters sound, and is a function of the target's shape, size, orientation, material properties (mass density, $\rho$, and sound speed $c$ ) and the acoustic wave number, $k$, of the incident wave $(k=2 \pi / \lambda$, where $\lambda$ is the acoustic wavelength). The scattering amplitude for sound scattering from any weak scatterer with a finite body of volume $V$ (such as the animals under investigation here, which have material properties that are similar to those of the surrounding medium) can be modeled in the far field using the Born approximation (Morse and Ingard, 1968). In fact, all formulations and measurements presented in this paper are for backscattering, in which case the backscattering amplitude, $f_{b s}$, is given by

$$
f_{b s}=\frac{k_{1}^{2}}{4 \pi} \iint_{V} \int\left(\gamma_{\kappa}-\gamma_{\rho}\right) e^{2 i \mathbf{k}_{2 i} \cdot \mathbf{r}_{v}} d V,
$$

where $k_{1}$ is the wave number of the incident sound $\left(k_{1}\right.$ $\left.=\left|\mathbf{k}_{1 i}\right|\right), \mathbf{k}_{2 i}$ is the wave vector of the incident sound evaluated in the interior of the volume, $\mathbf{r}_{v}$ is the position vector of any volume element, $\gamma_{\kappa}=\left(\kappa_{2}-\kappa_{1}\right) / \kappa_{1}=\left(1-g h^{2}\right) / g h^{2}, \gamma_{\rho}$ $=\left(\rho_{2}-\rho_{1}\right) / \rho_{2}=(1-g) / g$, and $g\left(=\rho_{2} / \rho_{1}\right)$ and $h$ $\left(=c_{2} / c_{1}\right)$ are the density and sound-speed contrasts, respectively. $\kappa_{1}\left(=\left(\rho_{1} c_{1}^{2}\right)^{-1}\right)$ and $\kappa_{2}\left(=\left(\rho_{2} c_{2}^{2}\right)^{-1}\right)$ are the compressibilities in the surrounding medium and body interior. It should be noted that the wave number in the exponent has been evaluated within the body interior ( $\mathbf{k}_{2 i}$ instead of $\mathbf{k}_{1 i}$ in the exponent). This modification to the Born approximation has been referred to as the distorted wave Born approximation (DWBA). Throughout this work, the subscript " 1 " indicates that the quantity referred to is evaluated in the surrounding medium, while the subscript " 2 " indicates that the quantity referred to is evaluated in the body interior. If the material properties are constant throughout the volume $\left(\gamma_{\kappa}\right.$ $-\gamma_{\rho}$ ) can be factored out of the integral, and, at very low frequencies, it is straightforward to show that $f_{b s}$ is proportional to $\mathrm{V}\left(f_{b s}=\left(k_{1}^{2} / 4 \pi\right)\left(\gamma_{\kappa}-\gamma_{\rho}\right) V\right)$.

For an elongated body with a circular cross section at every point along its lengthwise axis, Eq. (1) can be further simplified to a one-dimensional line integral (Stanton et al., 1998b), given by

$f_{b s}=\frac{k_{1}}{4} \int_{r_{p}}\left(\gamma_{\kappa}-\gamma_{\rho}\right) a \frac{J_{1}\left(2 k_{2} a \cos \beta_{\mathrm{tilt}}\right)}{\cos \beta_{\mathrm{tilt}}} e^{2 i \mathbf{k}_{2 i} \cdot \mathbf{r}_{p}}\left|\mathbf{d r}_{p}\right|$,

where $\mathbf{r}_{p}$ is the position vector of the body centerline, $a$ is the radius, and $\beta_{\text {tilt }}$ the local tilt angle of the body cross section relative to $\mathbf{k}_{1 i}$ at each point on the axis. In this case, $\left(\gamma_{k}\right.$ $\left.-\gamma_{\rho}\right), a$, and $\beta_{\text {tilt }}$ can vary along the length of the body, but are restricted to a constant value in any given cross section.

For a small number of cases it is possible, by making very restrictive, but simplifying, assumptions about the shape and material properties (e.g., fluid-filled sphere or ellipsoid), to obtain a closed-form mathematical expression for the backscattering amplitude. In general, however, particularly when the conditions are as complex as those of the decapod shrimp we are attempting to model here, it is necessary to solve for the backscattering amplitude numerically. 
The far-field backscattered energy is often expressed in terms of the target strength (TS) with units of decibel (dB) relative to $1 \mathrm{~m}^{2}$ (Urick, 1983), and is given by

$$
\mathrm{TS}=10 \log \sigma_{b s}=10 \log \left|f_{b s}\right|^{2},
$$

where $\sigma_{b s}=\left|f_{b s}\right|^{2}$ is the differential backscattering cross section, and differs from the often-used backscattering cross section $\sigma$ by a factor of $4 \pi\left(\sigma=4 \pi \sigma_{b s}\right)$. In order to compare scattering from objects of different sizes but similar proportions, TS is often normalized according to the square of some typical dimension, giving rise to the reduced target strength (RTS). For elongated zooplankton, of length $L$, the RTS is given by

$$
\mathrm{RTS}=10 \log \frac{\sigma_{b s}}{L^{2}}=10 \log \left|f_{b s}\right|^{2}-10 \log L^{2} .
$$

\section{B. Modeling considerations}

The most rigorous method of modeling the acoustic scattering from a complex body is to digitize the body shape and material properties within the body in increments significantly smaller than the wavelength. These high-resolution digitizations can then be used as input to a general 3D scattering model, such as that given by Eq. (1). Under most circumstances, measurements at a resolution of approximately $\lambda / 10$ to $\lambda / 20$ are required to accurately represent the animal's body, although the exact value may vary with the modeling approach (Stanton and Chu, 2000). This digitization criterion for the outer boundary shape is met for the range of frequencies used in these experiments when the animal shape is obtained from CT scans. However, if high-resolution CT scans are not available, it is possible to perform lower resolution 2D measurements of outer boundary shape, and use these digitizations as input to a simplified scattering model, such as that given by Eq. (2). As will be seen later, there are circumstances under which caution must be used when applying the line-integral model, although there are conditions when the results of the line-integral and volume-integral models are very similar. Comparison of the results obtained using these two approaches is one of the goals of this work.

High resolution digitizations of the material properties are also necessary to accurately evaluate $f_{b s}$. However, there are only a few studies in which the material properties of fluid-like zooplankton have been investigated (Greenlaw, 1977; Foote, 1990; Chu et al., 2000) and in these cases, only the average quantities were studied. There is little information available as to the spatial variability of these properties throughout the body interior (Yayanos et al., 1978; Foote, 1998). For most of the work presented here, $g$ and $h$ are held constant throughout the body interior, with values of $g=1.0357$ and $h=1.0279$ taken from Foote (1990), as measured for Euphausia superba.

\section{EXPERIMENTAL METHODS}

\section{A. Acoustic data acquisition}

The acoustic backscattering measurements involving individual decapod shrimp were made on ten specimens, but only data collected from animals that remained alive and in

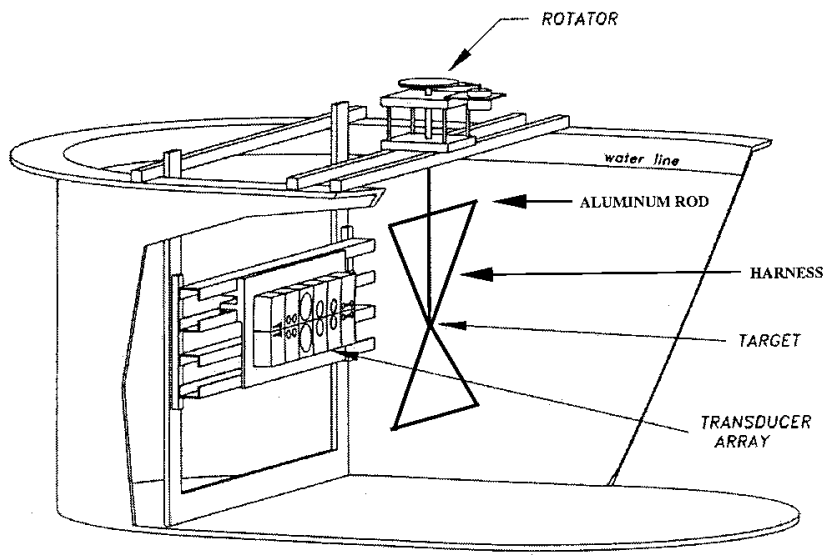

FIG. 1. Schematic of the experimental system used for acquiring acoustic backscattering data as a function of angle of orientation and frequency: tank, transducer array, stepper motor, and (acoustically transparent) harness system with horizontal aluminum rods used to tether the decapod shrimp.

good condition throughout the data acquisition process are considered here, namely the four animals numbered $6,8,9$, and 10 . The experimental setup was very similar to that described in Stanton et al. (2000), and only a brief overview of the relevant equipment and methods will be described here together with a description of the difference between the two setups. The data presented in this study were collected in 1996 in the acoustics laboratory at the Woods Hole Oceanographic Institution.

The experiments were performed in a 1.5-m-deep by 3.7-m-long and 2.4-m-wide tank filled with filtered seawater (Fig. 1). An array of transducer pairs was mounted in the tank facing horizontally. A combination of single-frequency and broadband transducers was employed to collect the acoustic backscattering data. The transducers within each pair were placed in a bistatic configuration, as close as possible to each other so as to approximate a monostatic configuration. Six pairs of powerful narrow-band (NB) transducers, emitting 200- $\mu$ s-long gated sine waves, were employed at the following frequencies: 50, 75, 120, 165, 200, and 258 kHz. In addition, two octave-bandwidth broadband (BB) transducers were also employed, emitting 200- $\mu$ s-long "chirp" (linear frequency modulated) signals with center frequencies at 250 and $500 \mathrm{kHz}$. The transmitted voltage time series, $v_{b s}^{T}(t)$, and the received voltage time series, or equivalently, the backscattered return echo from the animal, $v_{b s}^{R}(t)$, were stored on a personal computer for later analysis. One of the main advantages of using broadband transducers is that large amounts of information can be gathered over a wide frequency range, allowing both temporal and spectral data analysis techniques to be exploited, maximizing the information that can be extracted about the sound scattering process. However, a considerable drawback of employing broadband signals is that less energy can be applied per frequency bin.

The animals were tethered in a two-point acoustically transparent harness made of 59- $\mu \mathrm{m}$-diameter monofilament line (Fig. 1). This involved a loop around the thorax, and another line running through the midpoint of the abdomen [Fig. 2(a)]. The loop was loose enough to permit normal respiration and blood flow, but tight enough to prevent sub- 
(a)

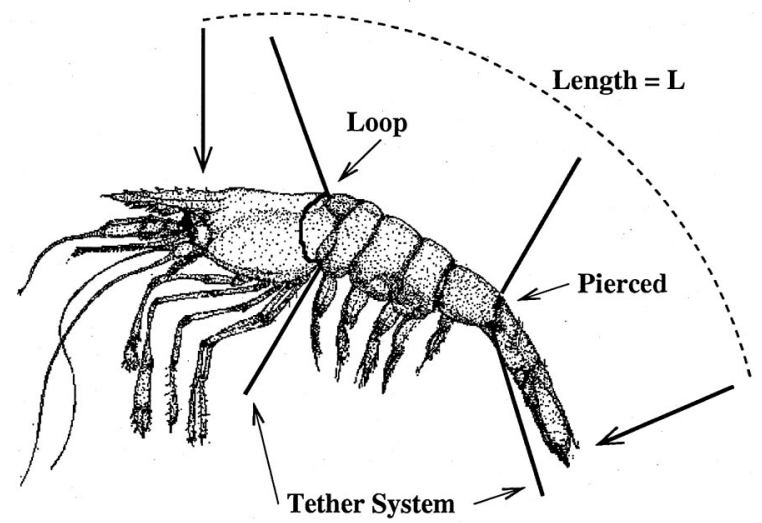

(b)

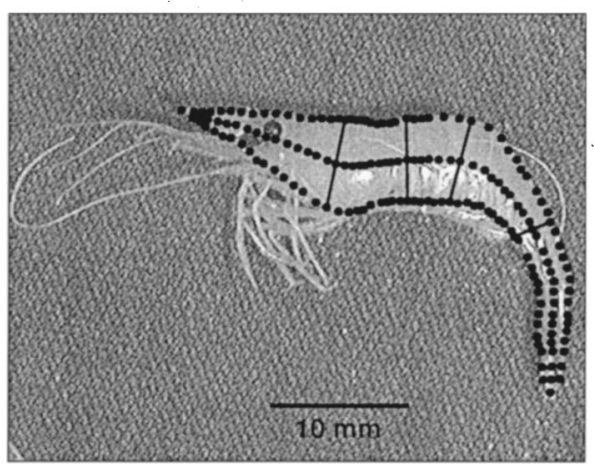

FIG. 2. (a) Schematic diagram of a decapod shrimp, showing the two-point tether. The animals were tethered with a loop about the thorax and were also pierced through the abdomen. The animal lengths (L) were measured from the photographic images. (b) The animal shape was digitized in $2 \mathrm{D}$ by obtaining measurements in the dorsal-ventral plane. The measurements are shown as black points along the outside contour of the animal. The calculated centerline is also shown (from which the length, L, was calculated), together with a selection of radii. For the scattering predictions made using the line-integral model, based on the 2D digitizations of animal shape, the animals were digitally straightened so that the shape of the animal more accurately reproduced the observed shape during the acoustic backscattering measurements. However, it was not necessary to digitally straighten the CT data since the animals were naturally straighter during the CT scanning process.

stantial movement. For the data reported here, the animals remained alive and in good condition throughout the data acquisition process. Tension was maintained in the harness by two horizontal aluminum rods. This kept the position and orientation of each animal stable. The animals were centered in the acoustic beam in the far field of the transducers at a range of $0.51 \mathrm{~m}$. A computer-controlled stepper motor rotated the animals in $1^{\circ}$ increments, in most cases through two full rotations $\left(720^{\circ}\right)$. The animals were insonofied with a single ping at each angle of orientation. Table I summarizes the frequencies and angles of orientations for which backscattering measurements were performed. Measurements of the animal lengths, radii, aspect ratios $(L / a)$, and wet weights are summarized in Table II.

Animals 6 and 8 were tethered such that the dorsalventral aspect was approximately in the vertical plane, with an out-of-plane tilt of $25^{\circ}-45^{\circ}$. In contrast, animals 9 and 10 were tethered such that the dorsal-ventral aspect of the animals was approximately in the horizontal plane, with an outof-plane tilt of $25^{\circ}-45^{\circ}$. There is some uncertainty in the tilt angle of the animals since there was no way to accurately
TABLE I. Range of orientations and frequencies used to obtain acoustic backscattering measurements.

\begin{tabular}{lcc}
\hline \hline Frequency $(\mathrm{kHz})$ & Animal no. & Range of orientations in $1^{\circ}$ increments \\
\hline 50 & $6,8,10$ & $0-720^{\circ}$ \\
75 & $6,8,10$ & $0-360^{\circ}$ \\
120 & $6,8,9,10$ & $0-720^{\circ}$ \\
160 & $6,8,9,10$ & $0-720^{\circ}$ \\
200 & $6,8,9,10$ & $0-720^{\circ}$ \\
258 & $6,8,9,10$ & $0-720^{\circ}$ \\
$250(\mathrm{BB})$ & $6,8,9,10$ & $0-720^{\circ}$ \\
$500(\mathrm{BB})$ & $6,8,9,10$ & $0-720^{\circ}$ \\
\hline \hline
\end{tabular}

quantify it and it also changed slightly as the animals were rotated.

The transducers were calibrated prior to the backscattering measurements following the procedure outlined in Stanton et al. (1998a). During the calibration, the receiver and transmitter were aimed at each other at a range $r_{\text {cal }}=0.69 \mathrm{~m}$. For each transducer pair, ten 200- $\mu$ s-long chirp signals were collected and averaged. The time series of the transmitted, $v_{\text {cal }}^{T}(t)$, and received, $v_{\text {cal }}^{R}(t)$, calibration voltages were stored for the purpose of postprocessing calibration of the scattering data. The background reverberation of the tank, without the animals present, was also measured for each transducer pair in the bistatic scattering configuration. This measurement involved averaging the background reverberation signals, for each set of transducers, over a large number of echoes. The average reverberation echo was stored on the data acquisition oscilloscope and subtracted in real time from the echoes obtained once the animals were placed in the tank. This procedure was repeated for each animal. Great care was taken throughout the data acquisition process to minimize the presence of bubbles on the tethers, since the acoustic signals from bubbles can greatly contaminate the acoustic returns of interest. For each acoustic return from each transducer pair

$$
f_{b s}=\frac{V_{b s}^{R}}{V_{\text {cal }}^{R}} \frac{V_{\text {cal }}^{T}}{V_{b s}^{T}} \frac{r_{b s}^{2}}{r_{\text {cal }}},
$$

where $V_{b s}^{R}, V_{b s}^{T}, V_{\mathrm{cal}}^{R}$, and $V_{\mathrm{cal}}^{T}$ are the absolute values of the Fourier transforms of $v_{b s}^{R}(t), v_{b s}^{T}(t), v_{\text {cal }}^{R}(t)$, and $v_{\text {cal }}^{T}(t)$, respectively. Prior to evaluating the Fourier transforms of the voltage time series, a bandpass filter was applied to the data, to reduce out-of-band noise.

\section{B. 2D measurements of animal shape}

For application of the line-integral model it was necessary to estimate the cylindrical radius of each animal at a number of points along the lengthwise axis. These measurements were performed by first taking $2 \mathrm{D}$ photographic images of the preserved animals in the dorsal-ventral plane. As mentioned earlier, one of the underlying assumptions of this model is that the animal cross section is circular. Consequently, it is sufficient to obtain 2D measurements of the animal radius in one plane at a number of points along the centerline axis. The animal shapes were digitized by collecting the coordinates of a number of points along their outer contours. Animal centerlines and radii were then calculated from these measurements. To obtain the coordinates and radii 
TABLE II. Animal lengths (defined in Fig. 2), average torso radii, wet weights, aspect ratio, number of data points along the lengthwise axis (including end points) taken from the 2D photographic images, and availability of acoustic and CT data. The average torso radii were calculated from the $2 \mathrm{D}$ photographic images by averaging over all the radii, excluding the ten points at each end of each animal.

\begin{tabular}{ccccccc}
\hline \hline Animal no. & $\begin{array}{c}\text { Length }(\mathrm{L}) \\
(\mathrm{mm})\end{array}$ & $\begin{array}{c}\text { Radius }(a) \\
(\mathrm{mm})\end{array}$ & $\begin{array}{c}\text { Wet weight } \\
(\mathrm{g})\end{array}$ & $\begin{array}{c}\text { Aspect ratio } \\
(L / a)\end{array}$ & $N$ & Acoustic: CT \\
\hline 6 & 30 & 1.5 & 0.17 & 20.5 & 49 & Yes:No \\
8 & 25 & 1.35 & 0.10 & 17.7 & 46 & Yes:No \\
9 & 25 & 1.6 & 0.10 & 15.6 & 45 & Yes:No \\
10 & 26 & 1.4 & 0.13 & 18.1 & 47 & Yes:No \\
11 & 39 & 2.2 & 0.48 & 17.9 & 57 & No:Yes \\
13 & 35 & 2.3 & 0.45 & 16.9 & 43 & No:Yes \\
14 & 30 & 1.65 & 0.19 & 18.2 & 41 & No:Yes \\
\hline \hline
\end{tabular}

of $N$ points along the centerline axis, including two end points, it is necessary to collect $(2 N-2)$ points along the outer contour of the animal (Table II). The result of this 2D digitization process is shown in Fig. 2(b) for animal 11. Since these measurements were performed in the dorsalventral plane of the animals, the results more closely represent a side view of the actual animal than a top view. Finally, since the digitization of animal shape was performed after the animals had been preserved, it was necessary to digitally straighten the animals so that they more closely represented the actual live animal shape.

\section{3D measurements of animal shape: CT scans}

High-resolution computerized tomography (CT) scans were performed for three decapod shrimp (animals 11, 13, and 14) at the Massachusetts Eye and Ear Hospital using a spiral CT scanner. Scans of animals 13 and 14 were performed with the animals live (but not in water), while animal 11 was scanned after recent preservation. The $\mathrm{CT}$ scans were used to evaluate the $3 \mathrm{D}$ volume integral with the integration performed within MATLAB (version 5.3). Details of this procedure are described below.

The CT scans were performed along the lengthwise axis of the animals, with a full set of slices obtained in a 1-2 s time interval. The separation, $d z$, between the slices was 100 $\mu \mathrm{m}$ for animals 13 and 14 , corresponding to the resolution limit of the CT scanner, while for animal 11 the separation between the slices was $200 \mu \mathrm{m}$. This resulted in $N_{z}$ slices per animal, which varied according to the animal length (Table III). A 3D image of animal 11 is shown in Fig. 3(a), together with a number of representative CT slices. The resolution of the CT data along the lengthwise axis of the animal, deter-

TABLE III. Animal number, number of CT slices $\left(N_{z}\right)$, number of pixels per CT slice $\left(N_{p} \times N_{p}\right)$, separation between CT slices $(d z)$, and pixel area within each slice. Note that the length of animal 13 as measured from the photographic images (Table II) is smaller than that of animal 11. However, there are more CT slices of animal 13 since more of the antennae were captured during this set of scans. These animal parts are not expected to contribute significantly to the scattering; the lengths used to calculate the RTS values are based on Table II.

\begin{tabular}{ccccc}
\hline \hline Animal no. & $N_{z}$ & $N_{p} \times N_{p}$ & $d z(\mu \mathrm{m})$ & Pixel area $(\mu \mathrm{m})^{2}$ \\
\hline 11 & 156 & $128 \times 128$ & 200 & $100 \times 100$ \\
13 & 399 & $100 \times 100$ & 100 & $104 \times 104$ \\
14 & 301 & $100 \times 100$ & 100 & $104 \times 104$ \\
\hline \hline
\end{tabular}

J. Acoust. Soc. Am., Vol. 111, No. 3, March 2002 mined by $d z$, and the resolution within any given slice, determined by the focus and zoom of the scanner, differ. However, for the CT data acquired during this study, both measures of resolution adequately meet the resolution criterion stated earlier, over the full range of acoustic wavelengths used for the data acquisition. For instance, at 500 $\mathrm{kHz}, 200 \mu \mathrm{m}$ corresponds to approximately $\lambda / 15$.

For each animal, the original CT slices contained 512 $\times 512$ pixels. For ease of manipulation and numerical efficiency, it was possible to crop each CT slice (referenced to a common pixel to maintain overall alignment) to contain $N_{p}$ $\times N_{p}$ pixels. Each CT slice was then converted to a binary
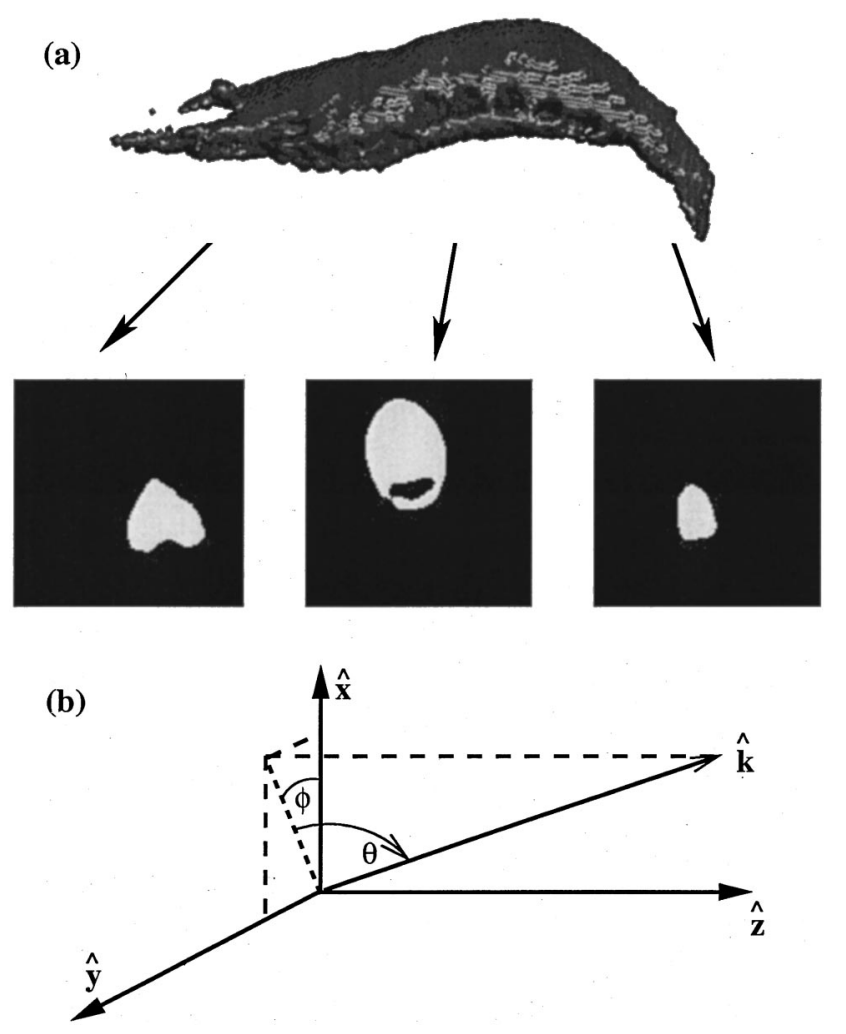

FIG. 3. (a) 3D image of animal 11 , together with three representative CT slices. (b) Scattering geometry used in the scattering models. The $\hat{z}$ axis is aligned along the lengthwise axis of the animal. Broadside incidence corresponds to, approximately, $\theta=0^{\circ}$ and $\theta=180^{\circ}$. Head-on incidence corresponds to $\theta=-90^{\circ}$, or equivalently, $\theta=270^{\circ}$. End-on incidence corresponds to, approximately, $\theta=90^{\circ}$. The $\phi=0^{\circ}$ and $180^{\circ}$ planes correspond to the dorsal and ventral sides of the animals, while the $\phi= \pm 90^{\circ}$ planes corresponds to the left and right sides of the animal. 
matrix, $\mathbf{M}^{l}$, of size $N_{p} \times N_{p}$, where $l$ runs from 1 to $N_{z}$. Matrix elements corresponding to pixels with an intensity above a certain threshold value contain ones and all others elements are zero. The matrices $\mathbf{M}^{l}$ were relatively insensitive to the value chosen for the threshold once it was set high enough to eliminate background diffraction effects. Thus, for each incident wave vector, the backscattering amplitude, $f_{b s}$, given as a volume-integral by Eq. (1), becomes

$$
\begin{aligned}
f_{b s}= & \frac{k_{1}^{2}}{4 \pi}\left(\gamma_{\kappa}-\gamma_{\rho}\right) \sum_{l=1}^{N_{z}} \sum_{j=1}^{N_{p}} \sum_{i=1}^{N_{p}} M_{i j}^{l} \\
& \times e^{2 i k_{2}\left(\cos \theta \cos \phi X_{i j}^{l}+\cos \theta \sin \phi Y_{i j}^{l}+\sin \theta Z_{i j}^{l}\right)} d V_{i j}^{l} .
\end{aligned}
$$

$X_{i j}^{l}, Y_{i j}^{1}$, and $Z_{i j}^{1}$ are the position vectors of each pixel, $d V_{i j}^{l}$ corresponds to the volume associated to each pixel, and the material properties have been assumed constant throughout the body interior. The direction of the incident (and backscattered) wave vector is $\hat{k}_{l i}=(\cos \theta \cos \phi \hat{x}+\cos \theta \sin \phi \hat{y}$ $+\sin \theta \hat{z}$ ), where the coordinate system is aligned with the CT scanner coordinate system [Fig. 3(b)]. Thus, the $\hat{z}$ direction is, approximately, along the lengthwise axis of the animals. Broadside incidence corresponds to $\theta=0^{\circ}$ or $\theta=180^{\circ}$, in some $\phi$ plane, while $\theta= \pm 90^{\circ}$ corresponds to end-on and head-on incidence, in any $\phi$ plane.

Simple variations in the material properties along the lengthwise axis of the animals can be incorporated into Eq. (6) by allowing the quantity $\left(\gamma_{\kappa}-\gamma_{\rho}\right)$ to vary between CT slices. Thus, Eq. (6) becomes

$$
\begin{aligned}
f_{b s}= & \frac{k_{1}^{2}}{4 \pi} \sum_{l=1}^{N_{z}} \sum_{j=1}^{N_{p}} \sum_{i=1}^{N_{p}}\left(M^{\prime}\right)_{i j}^{l} \\
& \times e^{2 i k_{2}\left(\cos \theta \cos \phi X_{i j}^{l}+\cos \theta \sin \phi Y_{i j}^{l}+\sin \theta Z_{i j}^{l}\right)} d V_{i j}^{l},
\end{aligned}
$$

where $\left(M^{\prime}\right)_{i j}^{l}=\left(\gamma_{\kappa}-\gamma_{\rho}\right)^{l} M_{i j}^{l}$, and $\left(\gamma_{k}-\gamma_{\rho}\right)^{l}$ has a single value within any given CT slice. This is only an approximate method of incorporating lengthwise variations in the material properties of the animals since it does not take into account the degree of animal bend.

Full 3D variation in the material properties can also be easily incorporated in a similar manner. In this case, $\left(M^{\prime}\right)_{i j}^{l}$ $=\left(\gamma_{\kappa}-\gamma_{\rho}\right)_{i j}^{l} M_{i j}^{l}$, where $\left(\gamma_{\kappa}-\gamma_{\rho}\right)_{i j}^{l}$ has a distinct value for each pixel. Finally, it is worth noting that it may be possible to extract density contrast values from the CT data, although it would be necessary first to calibrate the CT data.

\section{Averages}

In general, the scattering process is highly complex and can depend on many parameters simultaneously. Given the uncertainty in each parameter, predictions of a single scattering realization are often difficult to make. As a result, comparisons between predictions and data for single realizations are generally qualitative. Quantitative comparisons can be made for both the statistics of scattering and for averaged levels, where the averages can be over some distribution of parameters, such as a distribution of sizes or angles of orientation. There is also a more direct relationship between this approach and volume scattering data, such as that collected from aggregations involving a varied distribution of animal

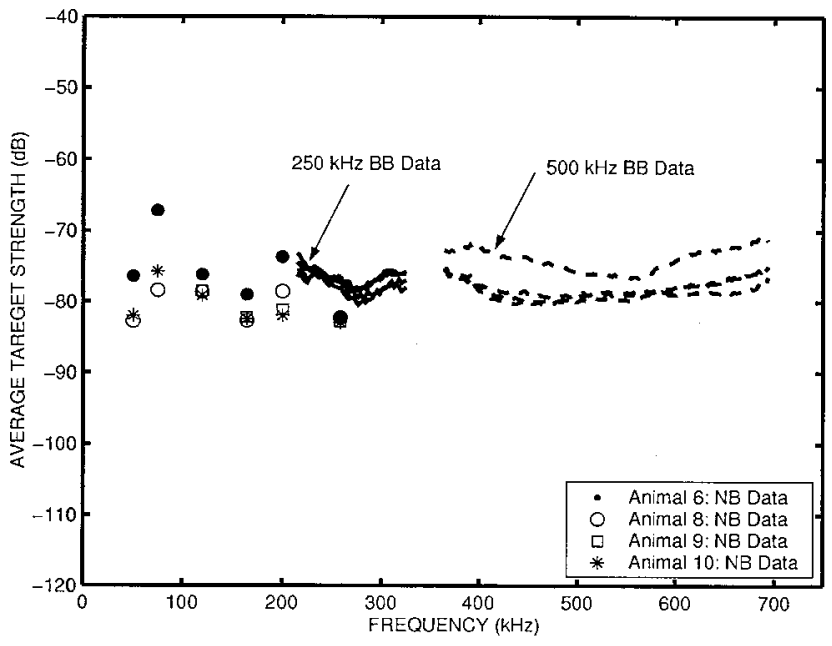

FIG. 4. TS averaged over orientation vs frequency for animals $6,8,9$, and 10. The four solid lines represent data obtained using the $250-\mathrm{kHz}$ broadband $(\mathrm{BB})$ transducer. The four dashed lines represent data obtained using the $500-\mathrm{kHz}$ broadband transducer. The symbols represent the average TS values obtained from the single-frequency, or narrow-band (NB), data. The averages are taken for all angles uniformly in one plane.

sizes and angles of orientation. It is possible to predict average scattering levels more accurately since the complex and stochastic structure of the scattering is smoothed during the averaging process. In this paper, the focus is on averages over orientation since the number of animals for which there are scattering data, and their size range, is relatively small.

At sufficiently high frequencies, acoustic returns from aggregations of zooplankton tend to add incoherently. Thus, average TS $\left(=10 \log \overline{\sigma_{b s}}\right)$ values were obtained by averaging the differential backscattering cross section, $\sigma_{b s}$, over all angles of orientation. Since there is little information available as to the natural orientation distributions of decapod shrimp, or any other elongated fluid-like animals, average TS values were calculated by assuming a uniform distribution of orientations from $0-360^{\circ}$.

The average TS values as a function of frequency were calculated for all four animals, for both single-frequency and broadband data, and these results are shown in Fig. 4. Once averages are taken, much of the fine structure observed in the data on a ping-by-ping basis is smoothed out. The TS values for animal 6 obtained from the $500-\mathrm{kHz}$ broadband transducer were approximately $6 \mathrm{~dB}$ higher than those obtained for the other animals. Although no errors were noted during the data acquisition process for this animal, it is hypothesized that this anomaly could be due to an error in an oscilloscope setting.

\section{MODEL PREDICTIONS}

\section{A. Comparison of TS predictions versus angle}

Though the TS values predicted by both models spanned a similar range for the frequencies and angles investigated, it was found that the differences between the predicted scattering levels were generally larger at angles close to end-on incidence than at angles close to broadside (Fig. 5), with the line-integral model predicting considerably higher values at angles close to end-on incidence. These differences were 

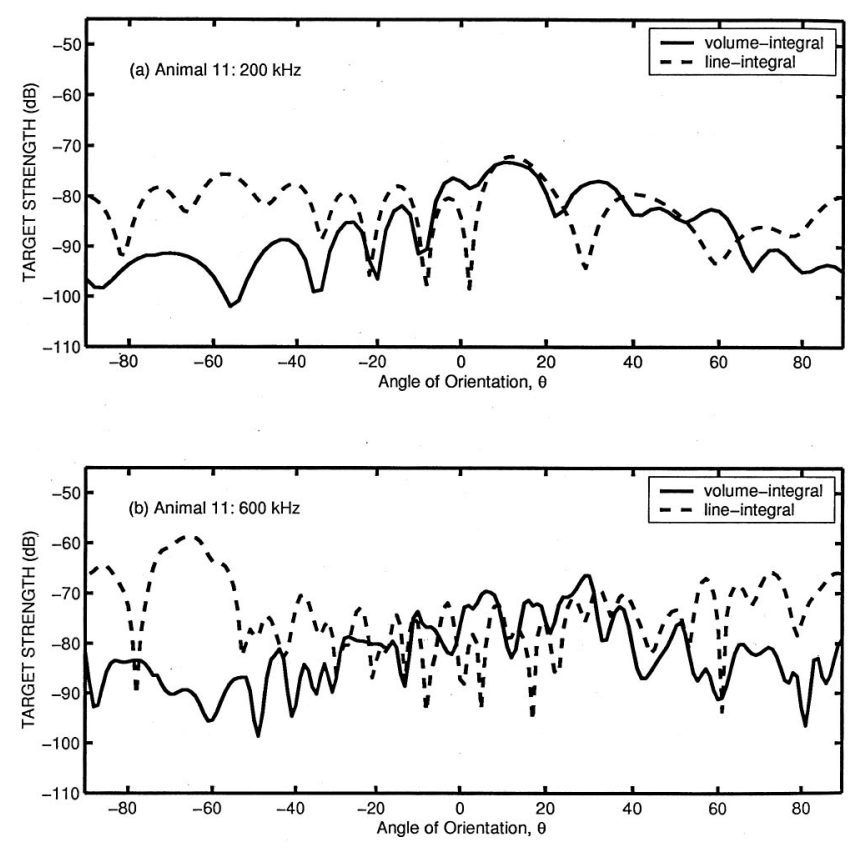

FIG. 5. Comparison of TS predictions for animal 11, in the $\phi=0^{\circ}$ plane (dorsal-ventral), based on the line-integrated (dashed line) and volumeintegral (solid lines) models. (a) $200 \mathrm{kHz}$ and (b) $600 \mathrm{kHz}$. The largest differences observed between the two models occur at angles close to head-on and end-on incidence $\left(\theta= \pm 90^{\circ}\right)$ where the line-integral predicts higher backscattering levels, particularly at higher frequencies.

accentuated at the higher frequencies [Fig. 5(b)]. For ease of intermodel comparison, most scattering predictions were performed in the $\phi=0^{\circ}$ plane. However, predictions were also made in the $\phi=45^{\circ}$ and $\phi=90^{\circ}$ planes, with trends in the results similar to those found for the $\phi=0^{\circ}$ plane.

\section{B. Comparison of average TS predictions}

The average TS was initially calculated for averages taken over all angles of orientation uniformly distributed in the $\phi=0^{\circ}$ plane [Fig. 6(a)]. The average TS values predicted by both models agree closely at the lower frequencies, but the agreement deteriorates with increasing frequency. At 700 $\mathrm{kHz}$, the line-integral prediction lies almost $10 \mathrm{~dB}$ higher than the volume-integral prediction. At high frequencies, the volume-integral model predicts that the average TS attains a relatively constant value, whereas the line-integral model predicts that the average target strength slowly increases with increasing frequency, over the frequency range investigated. Average TS values were then calculated with the $\pm 25^{\circ}$ centered about end-on incidence excluded [Fig. 6(b)]. In this case, both models predict very similar average TS levels. It can be seen that the differences between the model predictions are generally within several $\mathrm{dB}$ at most frequencies investigated. It is apparent from Figs. 5 and 6 that at higher frequencies the line-integral model is predicting significantly higher average TS levels when the averages include angles close to end-on incidence. This trend was observed for all $\phi$ planes investigated.
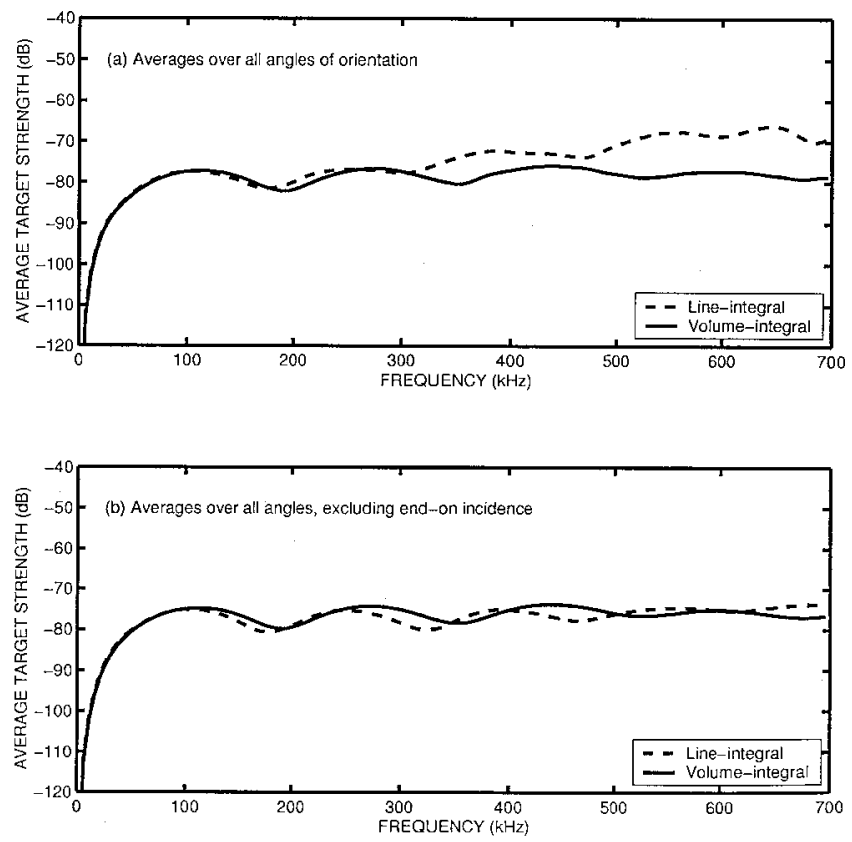

FIG. 6. Comparison of TS averaged over orientation vs frequency predicted by the line-integral (dashed lines) and the volume-integral (solid lines) models for animal 11 in the $\phi=0^{\circ}$ plane (dorsal-ventral). (a) Averages performed over all angles of orientation uniformly. (b) Averages performed uniformly over all angles of orientation excluding the range of angles $\pm 25^{\circ}$ centered on end-on incidence.

\section{Comparison of average RTS predictions for different animals}

The differences in the average RTS values obtained using the volume-integral model, for the three animals for which CT scans were available, are illustrated in Fig. 7. The average RTS values are plotted as a function of the dimensionless product $k a$ so as to account for the different radii of the animals. (The values of $a$ used to evaluate $k a$ are those presented in Table II.) It can be seen that the structure of the

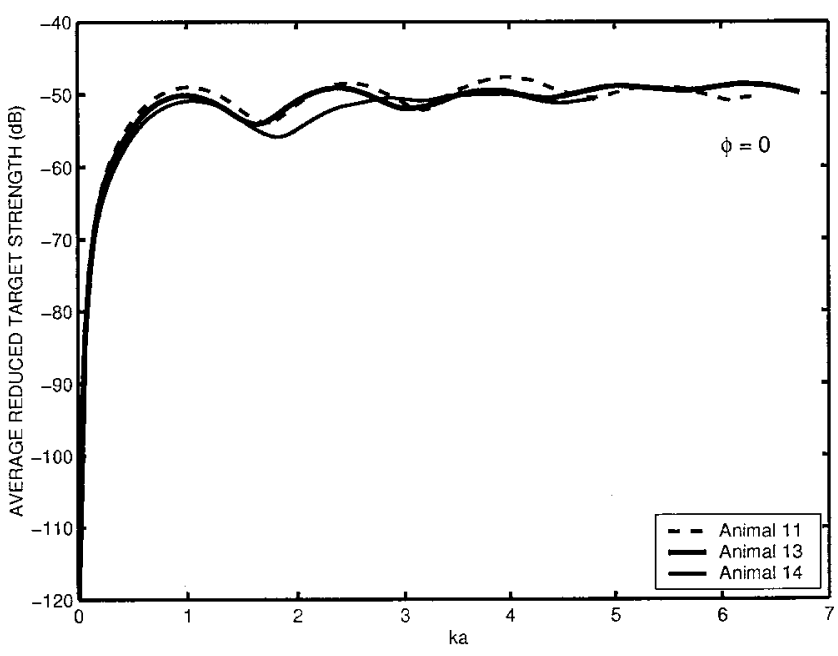

FIG. 7. Comparison of RTS averaged over orientation vs $k a$ predicted by the volume-integral model for animals 11 (dashed line), 13 (thick solid line), and 14 (thin solid line), in the $\phi=0^{\circ}$ plane (dorsal-ventral). The average torso radii, calculated from the $2 \mathrm{D}$ animal measurements (Table II), were used to evaluate $k a$. 


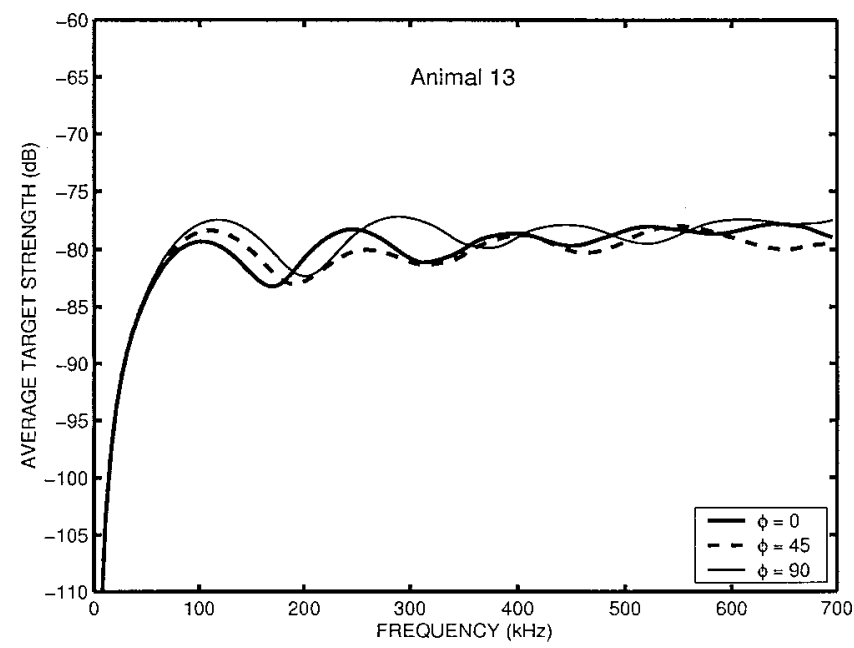

FIG. 8. Comparison of TS averaged over orientation vs frequency predicted by the volume-integral model for animal 13 in three different $\phi$ planes. The $\phi=0^{\circ}$ plane corresponds to the dorsal side of the animal, while $\phi=90^{\circ}$ corresponds to the left side of the animal.

average RTS is very similar for all three animals, and the transition from Rayleigh to geometric scattering occurs at similar $k a$ values.

\section{Comparison of average TS predictions for different $\phi$ planes}

To illustrate that the conclusions drawn above are not dependent on the $\phi$ plane chosen, average TS values for animal 13, calculated using the volume-integral model with $\phi=0^{\circ}, \phi=45^{\circ}$, and $\phi=90^{\circ}$, are shown in Fig. 8. It can be seen that the average TS values in the different planes differ by no more than a few $\mathrm{dB}$. There is a shift in the location of the first peak and null which is probably related to the fact that the average width of the animal is different in different $\phi$ planes.

\section{E. Variations in material properties}

The effect on backscattering due to changes in material properties was also briefly investigated. Two approaches were taken. The first approach involved studying the effects of changes in the values of the material properties, assuming homogeneous values throughout the body interior. The change in TS due to changes in the overall average levels of $g$ and $h$ is given by

$$
\Delta \mathrm{TS}=\mathrm{TS}^{(2)}-\mathrm{TS}^{(1)}=20 \log \frac{\left(\gamma_{\kappa}-\gamma_{\rho}\right)^{(2)}}{\left(\gamma_{\kappa}-\gamma_{\rho}\right)^{(1)}},
$$

where $\mathrm{TS}^{(1)}$ and $\mathrm{TS}^{(2)}$ are the target strength values obtained for (otherwise identical) animals with average material properties given by $\left(\gamma_{\kappa}-\gamma_{\rho}\right)^{(1)}$ and $\left(\gamma_{\kappa}-\gamma_{\rho}\right)^{(2)}$, respectively.

In the second approach, following the work by Stanton and Chu (2000), two different material property profiles were investigated, chosen to vary along the lengthwise axis of the animal only. Although it is possible that the actual material properties will vary in all directions, not just along the lengthwise axis, we limit this investigation to this particular scenario due to the large degree of speculation involved. In addition, since we are only illustrating the point that small

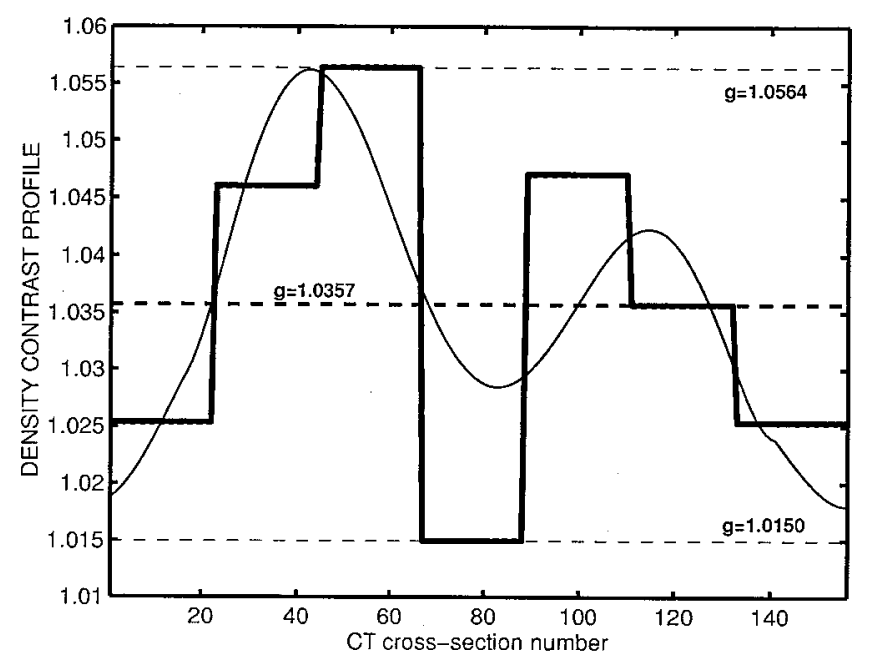

FIG. 9. Material property profiles. Three different uniform values of $g$ were investigated (dashed lines): $g=1.0564, g=1.0357, g=1.015$. The thick solid line represents the profile with seven segments with a mean value of $g=1.0357$. The thin solid line represents the smoothly varying profile, with the mean value also kept at $g=1.0357$. The material profiles only varied along the lengthwise axis of the animal.

changes in the material properties can cause large changes in the scattering, this study is limited to variations in the density contrast, $g$, keeping $h$ constant at all times $(h$ $=1.0279$ ). When dealing with inhomogeneous material properties, Eq. (8) is no longer applicable and the full solution for $f_{b s}$ must be calculated before averages can be taken. Certainly, due to the lack of information available, this work can only be viewed as exploratory in nature.

The two material property profiles investigated involved
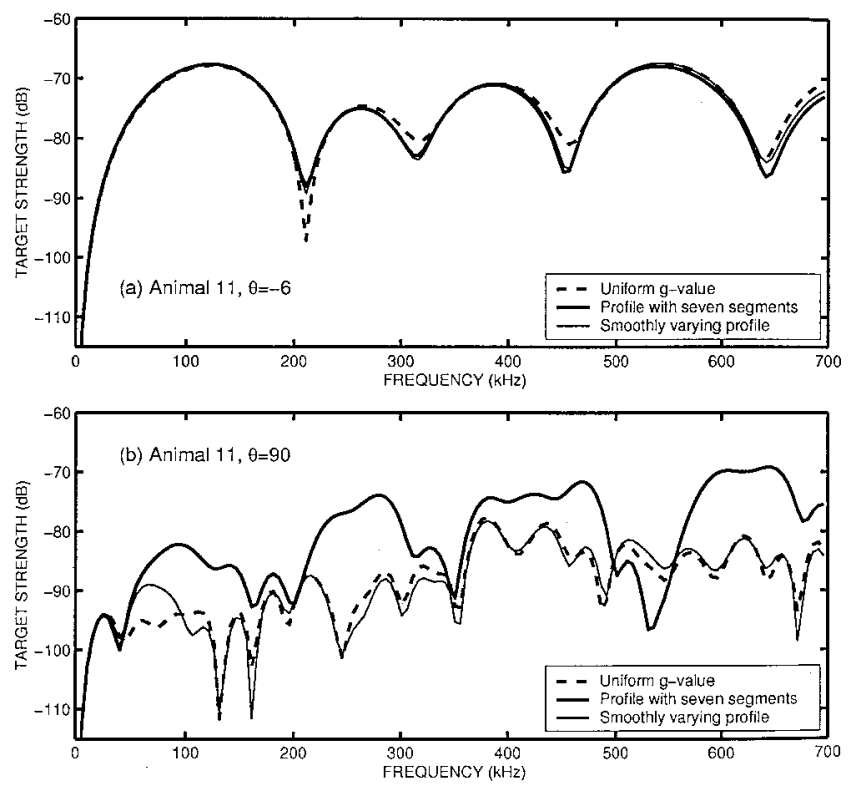

FIG. 10. Effects on TS due to changes in material property profiles. The predictions shown here are for a single realization based on the volumeintegral model for animal 11. (a) TS vs frequency for $\theta=-6^{\circ}$ and $\phi=0^{\circ}$ ( $\sim$ broadside). (b) TS vs frequency for $\theta=90^{\circ}$ and $\phi=0^{\circ}$ ( $\sim$ end-on). The thick dashed lines correspond to a uniform value of $g=1.0357$, the thick solid lines correspond to the segmented profile, and the thin solid line correspond to the smoothly varying profile [almost indistinguishable from the thick solid line in panel (a)]. The sound-speed contrast was held constant with a value of $h=1.0279$ for all curves. 


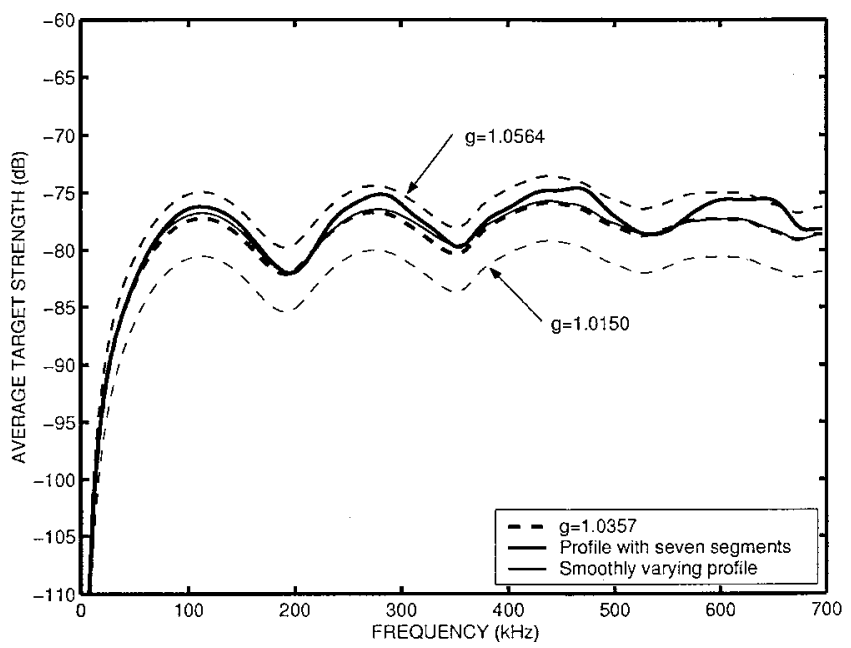

FIG. 11. Effects on TS, averaged over orientation due to changes in the material property profiles. The predictions shown here are based on the volume-integratal model for animal 11. The thin dashed lines correspond to uniform values of $g=1.0564$ (highest TS values) and $g=1.015$ (lowest TS values), respectively. The thick dashed line corresponds to a uniform value of $g=1.0357$, the thick solid line corresponds to the segmented profile, and the thin solid line corresponds to the smoothly varying profile. The sound-speed contrast was held constant with a value of $h=1.0279$ for all curves.
(1) a profile with seven segments and (2) a smoothly varying profile. These profiles are illustrated in Fig. 9. Each of these profiles contained $N_{z}$ points, equal to the number of CT slices. This approach was chosen since incorporation of these profiles into the DWBA-based volume-integral model was then straightforward, using Eq. (7), as outlined in Sec. III C. The segmented profile was chosen to have seven equal length segments, each with a constant value of $g$. The value assigned to $g$ in each segment was chosen randomly within the limits of $1.015 \leqslant g \leqslant 1.0564$, subject to the constraint that the average value of $g$ remained at 1.0357 . The smoothly varying profile was chosen such that it was varied, approximately, between the same constraints, with the same average value for $g$.

The effect on TS due to the different material property profiles was calculated for animal 11 at two specific angles of orientations [Figs. 10(a) and (b)]. The two scattering angles investigated were (a) $\theta=-6^{\circ}$ (close to broadside), and (b) $\theta=90^{\circ}$ (close to end-on incidence). $\theta=-6^{\circ}$ was chosen instead of $0^{\circ}$ since the peaks and nulls were more pronounced at this angle. Average TS, where the average is over all values of $\theta$ uniformly from 0 to $360^{\circ}$ in the $\phi=0^{\circ}$ plane, for the two material property profiles is shown in Fig. 11. The effect on backscattering when using the profile with seven segments is highly dependent on angle, and can be very large at angles close to end-on incidence. This is a good
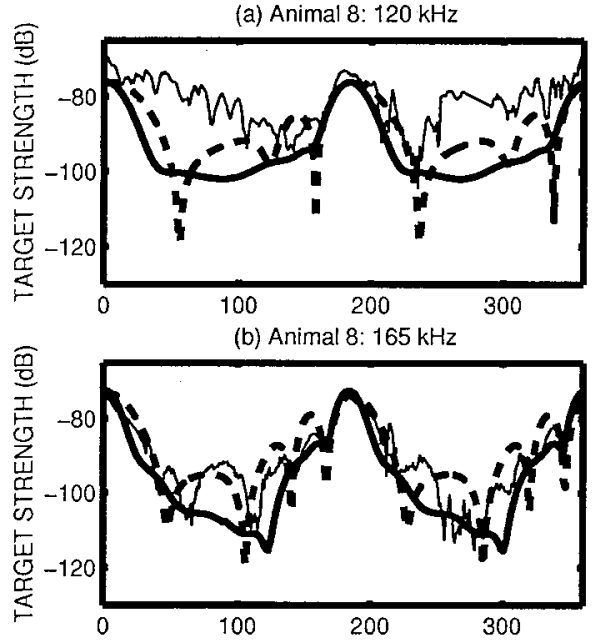

(c) Animal 8: $200 \mathrm{kHz}$
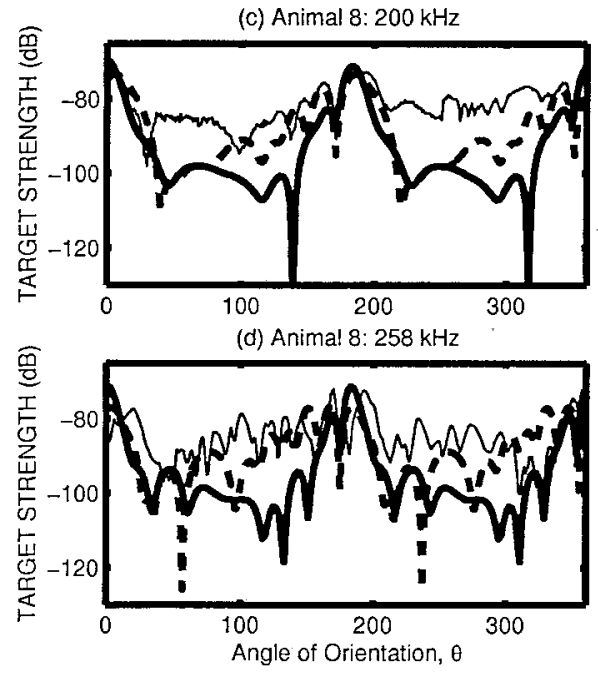

(e) Animal 9: $120 \mathrm{kHz}$
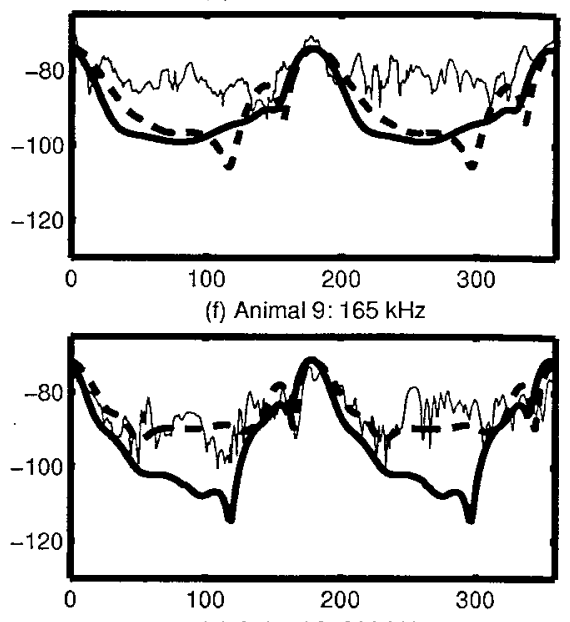

(g) Animal 9:200 kHz
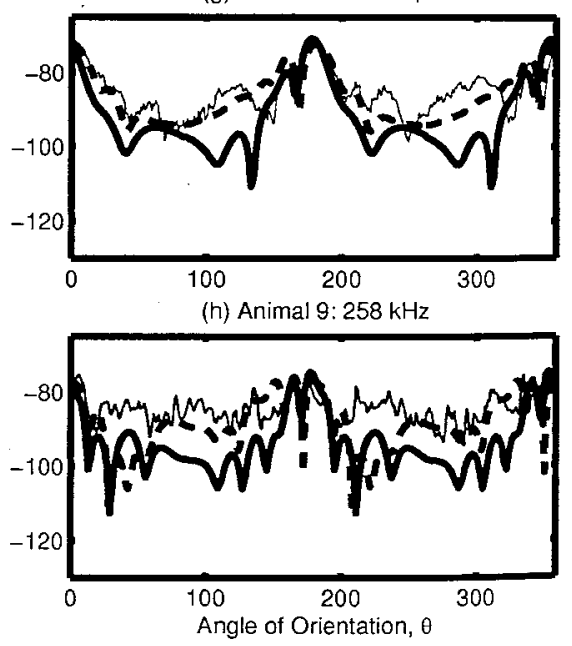

FIG. 12. TS vs $\theta$ for animals 8 (a) $-(d)$ and 9 (e)-(h) at $120 \mathrm{kHz}$ (a), (e), 165 $\mathrm{kHz}$ (b), (f), $200 \mathrm{kHz}$ (c), (g), and 258 $\mathrm{kHz}(\mathrm{d})$, (h). The dashed lines correspond to line-integral predictions, the thick solid lines correspond to volume-integral predictions, and the thin lines correspond to data. Broadside incidence corresponds to, approximately, $\theta=0^{\circ}$ and $180^{\circ}$. End-on scattering corresponds to $\theta=90^{\circ}$, and head-on scattering corresponds to $\theta=270^{\circ}$. The volume-integral predictions are based on the CT scans for animal 14, scaled to the size of animals 8 and 9 , respectively. To better match the observed animal orientation during the data acquisition, the line-integral predictions with the 2D measurements were performed with a $30^{\circ}$ out-of-plane tilt. However, the volume-integral predictions shown here, based on CT scans for animal 14, do not include any out-of-plane tilt. It is possible that the volume-integral predictions would better match the data if the dependence on tilt was further investigated. 

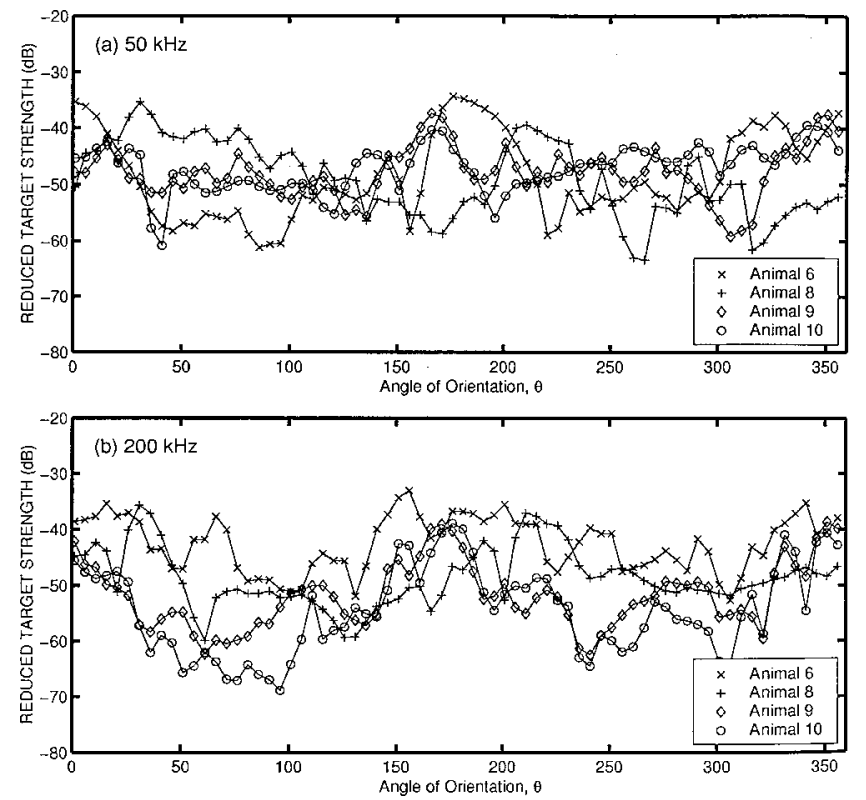

FIG. 13. Scatter plots of RTS vs $\theta$ for animals $6,8,9$, and 10 at (a) $50 \mathrm{kHz}$ and (b) $200 \mathrm{kHz}$. For all animals, broadside incidence corresponds to, approximately, $\theta=0^{\circ}$ and $180^{\circ}$. End-on and head-on incidence correspond to $\theta=90^{\circ}$ and $\theta=270^{\circ}$, respectively. For animals 6 and 8 , all data were collected with the dorsal-ventral aspects vertical (i.e., scattering in the sideaspect plane), while for animals 9 and 10 the dorsal-ventral aspect was horizontal. There was some degree of out-of-plane tilt for all animals, but typically $\leqslant 45^{\circ}$. This figure is included to illustrate the variability in the data.

illustration of the heightened sensitivity of the scattering process to small inhomogeneities at angles close to end-on incidence. This dependence, however, almost completely disappears when averages over all angles of orientation are taken. Also included in Fig. 11 is the TS for different constant values of $g: g=1.015, g=1.0357$, and $g=1.0564$.
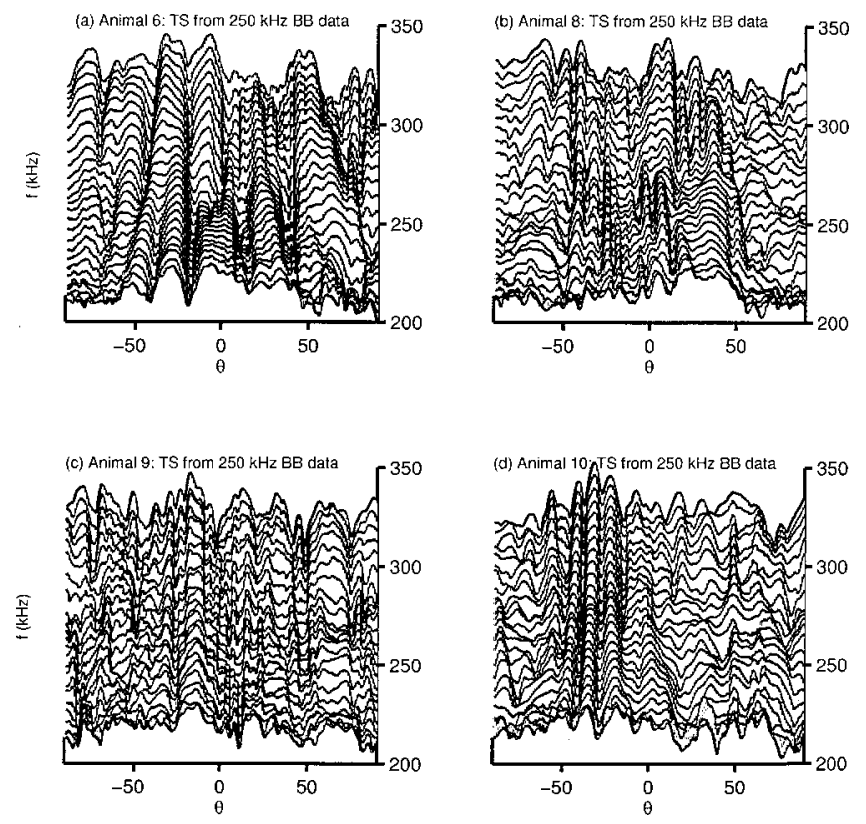

FIG. 14. TS vs frequency and $\theta$ obtained from the $250-\mathrm{kHz}$ broadband transducer for (a) animal 6; (b) animal 8; (c) animal 9; and (d) animal 10. For clarity, only the first $180^{\circ}$ of the data are shown here. The data have been averaged over a small frequency band to reduce the high levels of variability.
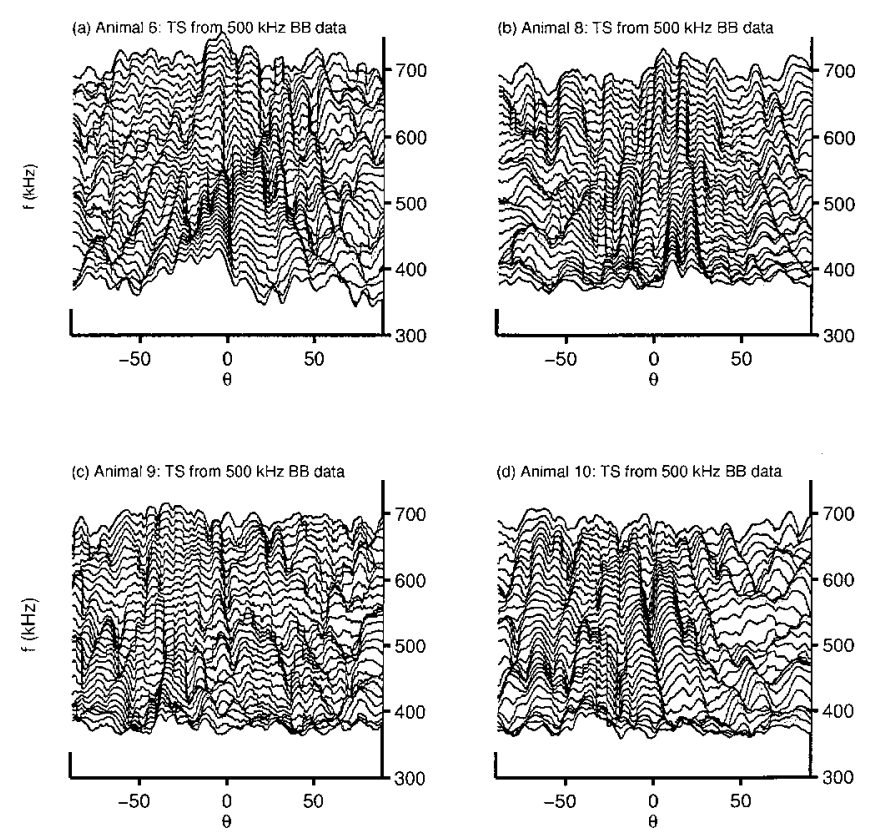

FIG. 15. TS vs frequency and $\theta$ obtained from the $500-\mathrm{kHz}$ broadband transducer for (a) animal 6; (b) animal 8; (c) animal 9; and (d) animal 10. For clarity, only the first $180^{\circ}$ of the data are shown here. The data have been averaged over a small frequency band to reduce the high levels of variability.

It is well known that scattering is highly sensitive to small changes in the average values of the material properties, in agreement with the modeling predictions presented here. Furthermore, it appears that the effects on backscattering (before average TS values are calculated) due to changes in the material property profiles have a relatively strong dependence on orientation, with the largest effects occurring at angles of orientation close to end-on incidence. Once average TS values are calculated, however, the differences in the scattering between the different material property profiles were almost completely eliminated. This is further evidence that reproducing scattering on a ping-by-ping basis is more difficult than predicting average quantities.

\section{MODEL AND DATA COMPARISONS}

\section{A. Ping-by-ping}

For all animals, it was found that the acoustic backscattering data were strongly dependent on both frequency and angle of orientation. To illustrate this, TS as a function of angle of orientation for animal 8 and 9 are shown in Fig. 12 at a selection of single frequencies. For comparison, predictions made with the line- and volume-integral models are also included. Since CT scans were not available for animals 8 and 9 as input into the volume-integral model, the CT data for animal 14 were scaled to the same aspect ratio as that of animals 8 and 9 . It can be seen that the orientation dependence of the backscattering increases with increasing frequency, and the agreement between both the line- and volume-integral predictions and the data is relatively good at angles close to broadside. However, the agreement is not as good at other angles, with differences of up to $20 \mathrm{~dB}$ observed at $120 \mathrm{kHz}$ for animal 9 between both models and the data. The general agreement between the line- and volume- 
integral predictions is also better close to broadside than at other angles. These observations indicate that the level of sophistication of the model is less critical at angles close to broadside. As will be discussed later, at these lower frequencies the line-integral model is expected to reproduce the data to a level of accuracy comparable to the volume-integral model. It was also found that, for all animals, the data were generally reproducible over the two full rotations, though the variability in the backscattering between the different animals at any particular frequency was significant (Fig. 13).

The broadband data were generally highly variable on a frequency-by-frequency and angle-by-angle basis, exhibiting much fine structure, particularly at the higher frequencies. To illustrate this variability, TS values over a wide range of frequencies and angles of orientation are shown in Figs. 14 and 15 , corresponding to data acquired with the 250- and $500-\mathrm{kHz}$ broadband transducers, respectively. To eliminate some of this variability and allow the prominent features in the data to be more easily observed, the data were averaged over a narrow range of frequencies. However, it can be seen that there is still a strong dependence on the frequency, the animal number, and the angle of orientation.

\section{B. Averages}

Given the challenges associated with comparisons of data and model predictions on a ping-by-ping basis, we also assess the model performance based on comparisons of averages taken over a uniform distribution of orientations. The predicted average RTS values for animal 11 are compared to the acoustic data obtained for animals 8 and 10, which had similar aspect ratios, in Fig. 16. The predictions based on both the line- and volume-integral models are included. Also included are data taken from Fig. 7 from Stanton et al. (1993b). These data were obtained for backscattering from aggregations of live tethered decapod shrimp (the same species as that used in the present study) over a wide range of frequencies from $50 \mathrm{kHz}$ to $1.2 \mathrm{MHz}$. Stanton et al. (1993b) also compared these data to a ray-based bent cylinder model in which averages over length and a uniform distribution of orientations were taken.

The line- and volume-integral models were used to make predictions for two different sets of $g$ and $h$ values [Figs. 16(a) and (b)]. Predictions made using $g=1.0357$ and $h=1.0279$ are presented in Fig. 16(a). These values of $g$ and $h$ were determined by Foote (1990) for Euphausia superba, and have been used in the literature for common shore shrimp. These values have been used for most of the predictions presented in this paper. However, Stanton et al. (1993b) found better agreement with the data when $g=h=1.06$ were used to make predictions with the ray-based model. The lineand volume-integral predictions using $g=h=1.06$ are shown in Fig. 16(b). Recent measurements of the material properties of decapod shrimp have been performed by Chu et al. (2000), resulting in values for $g(=1.043)$ and $h(=1.0649$ to 1.0736) that are close to the range of values investigated in this study and by Stanton et al. (1993b).

It can be seen that, for a particular choice of $g$ and $h$ both the line- and volume-integral models predict similar average RTS values for low $k a$ values. However, at higher $k a$
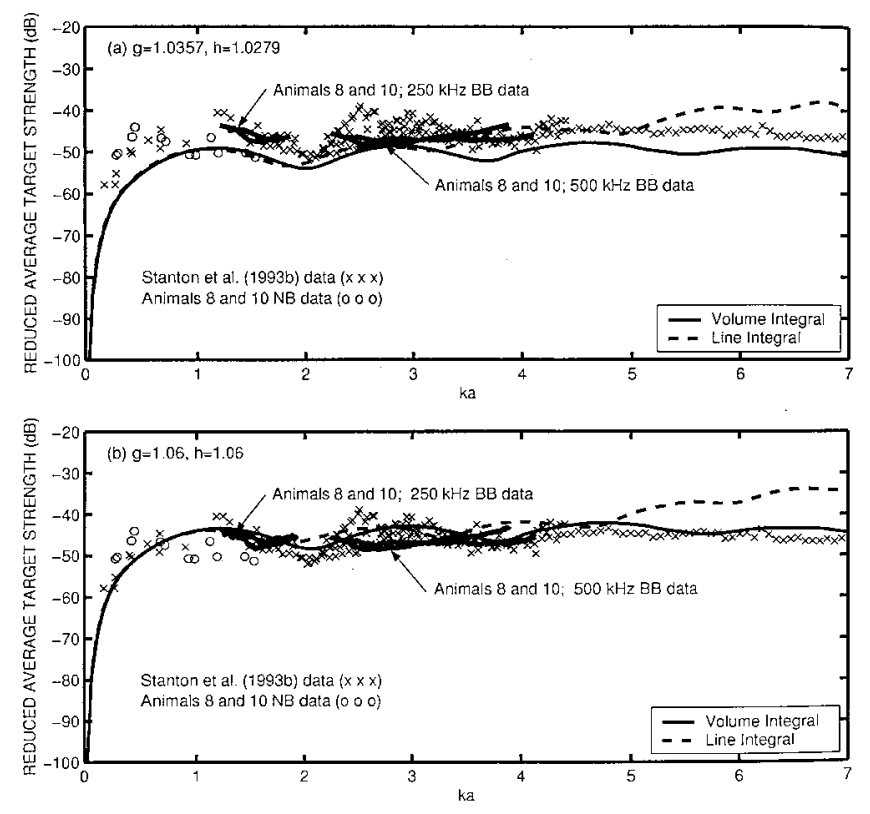

FIG. 16. Comparison of predicted and measured average RTS vs $k a$. In both panels, the thick solid lines correspond to the $250-\mathrm{kHz}$ and $500-\mathrm{kHz}$ broadband (BB) data obtained for animals 8 and 10 . The open circles correspond to the data obtained for animal 8 and 10 from the single-frequency, or narrow-band (NB), transducers. Data taken over a broad frequency range $(50 \mathrm{kHz}-1.2 \mathrm{MHz})$ for live tethered decapod shrimp aggregations [Fig. 7 from Stanton et al. (1993b)] are also included (crosses). This is the same species of animal as used throughout the current study. The dashed line corresponds to predictions for animal 11 based on the line-integral model, while the solid line corresponds to predictions based on the volume-integral scattering model. Animals 8,10 , and 11 had similar aspect ratios. Two different sets of values for $g$ and $h$ were investigated. (a) $g=1.0357$ and $h=1.0279$. These values are taken from Foote (1990) and are commonly used in the literature. (b) $g=h=1.06$. These values were used in Fig. 7 of Stanton et al. (1993b) to compare the predictions of an averaged ray-based model to the data included here. The agreement between the data and the models is better when $g=h=1.06$, particularly at low frequencies and close to the first null, which occurs at approximately $k a=2$. In addition, the volume-integral model also reproduces the location of a second null apparent in the data at approximately $k a=3.8$. The exact position of the nulls is very sensitive to the value used for the mean radius. The values for the mean radii for each animal shown in Table II were calculated from the 2D photographic images using all the radii except the ten points close to each end of the animal. However, the difference between the mean radius calculated this way and the maximum radius can be as large as $30 \%$. In fact, it was found that the best agreement between the model predictions (based on animal 11) and the Stanton et al. (1993b) data, in terms of the location of the first two nulls, occurred when the calculation of the mean radii included only the 10 points closest to the center of the animal, which is the dominant source of scattering.

values the line-integral model predicts significantly higher values for the average RTS than the volume-integral model, with difference between the two models of almost $10 \mathrm{~dB}$ at $k a=7$. As was observed by Stanton et al. (1993b), the agreement with the data is better when $g=h=1.06$ [Fig. 16(b)]. With these values of $g$ and $h$, the predicted average RTS values based on the volume-integral model for animal 11 generally agree well with the data over the range of $k a$ values investigated here.

It should be noted that the positions of the first two dips and nulls predicted by the models is highly sensitive to the value used for the mean radius. The mean radii shown in Table II were calculated from the 2D photographic images using all the measurements of radii along the lengthwise axis 
of the animals, excluding the ten points at each end of each animal (since the animals were strongly tapered there). However, these mean radii differed by as much as $30 \%$ from the maximum radius measured for each animal. It was found here that the positions of the first two nulls observed in Stanton et al. (1993b) data could be best reproduced by using the volume-integral model with a mean radius calculated from the 10 points centered around the location of the maximum radius (Chu et al., 1992). That is, the mean radius was calculated using the main part of the body only (i.e., the thorax section), which is similar to the mean radius used by Stanton et al. (1993b). For animal 11 this resulted in a mean thorax radius of $2.5 \mathrm{~mm}$ instead of $2.2 \mathrm{~mm}$ (an increase of approximately 14\%). With this value of mean radius, it can be seen that the position of the first two nulls in the Stanton et al. (1993b) data, at $k a=2$ and $k a=3.8$, are reproduced very well by the volume-integral model for animal 11 .

Just as we have observed here for predictions based on volume-integral model, Stanton et al. (1993b) also found good agreement between their ray-based predictions and the data, in terms of the locations of the first two nulls at $k a$ $=2$ and $k a=3.8$. However, the ray-based model predicted a deeper null at $k a=2$ than was observed in the data, with a difference between the averaged RTS values at the locations of the first peak and first null of approximately $12 \mathrm{~dB}$. The difference between the averaged RTS values at the location of the first peak and first null is approximately $5 \mathrm{~dB}$ for the volume-integral model which is in better agreement with the data. In fact, it can be seen by comparing the predictions made by Stanton et al. (1993b) for the ray-based model to the volume-integral model predictions shown here, that the oscillations in the average RTS versus $k a$ are more accentuated for the ray-based model over the entire $k a$ range investigated.

\section{DISCUSSION}

One of the main findings of this modeling study is that scattering from elongated fluid-like zooplankton at angles close to end-on incidence is significantly more sensitive to small changes in material properties and roughness, than scattering at angles close to broadside. Furthermore, this sensitivity is accentuated with increasing frequency. As a result, it is correspondingly harder to accurately predict scattering at angles close to end-on incidence.

At angles close to broadside, much of the structure observed in the target strength as a function of frequency, e.g., position and number of the peaks and nulls in the frequency spectra, can be relatively well accounted for by considering a simple two-ray model (Stanton et al., 1993a, 1993b; Stanton and Chu, 2000). For weak scatterers a large fraction of the incident energy is transmitted through the front interface. Consequently, constructive and destructive interference between the two primary rays that scatter from the front and back interfaces is principally responsible for the observed peaks and nulls. The phase difference between these two primary rays is determined by parameters such as the frequency and animal radius. Effects due to scattering from volume inhomogeneities and surface roughness are apparently small relative to the large primary returns from the front and back interfaces, explaining the relative lack of sensitivity of scattering at broadside incidence, as well as explaining the agreement observed between the different scattering models.

In contrast, at angles close to end-on incidence, the scattering is not dominated by two large returns from the front and back interfaces, and small-scale variability such as surface roughness and volume inhomogeneities play a more significant role. Thus, to accurately model scattering at angles close to end-on incidence it is necessary to have a more detailed knowledge of the small-scale variability. It is now possible to understand why predictions based on the lineintegral model do not agree with predictions based on the volume-integral scattering model at angles close to end-on incidence. There are a number of assumptions underlying the line-integral model. A key aspect of this formulation is that the animals are assumed to have a circular cross section at every point along their lengthwise axis. This assumption of cylindrical symmetry artificially introduces elevated roughness levels, through the creation of facets, which will be most highly accentuated at angles close to end-on incidence. This problem has been identified by Stanton and Chu (2000), and results in increased scattering levels due to scattering from these facets. Clearly, this problem is magnified with increasing frequency as it becomes increasingly harder to satisfy the $\lambda / 10-\lambda / 20$ resolution criterion mentioned earlier. Although it may be possible to reduce the effect of facets by increasing the resolution of the $2 \mathrm{D}$ measurements, it is an intrinsic problem associated with this model and cannot be eliminated entirely. Stanton and Chu (2000) also found that the problem could be reduced by smoothing the surface roughness profiles. However, the procedure was necessarily subjective. The more rigorous method to eliminate the problem of facets is to obtain 3D digitizations of animal shape and roughness, as was done in this study using highresolution CT scans. Finally, it should be noted that although predictions based on the volume-integral model are computationally more intensive than those based on the lineintegral model, the difficulty involved in obtaining 3D measurements of animal shape, and incorporating these measurements into the model, is not significantly greater than for the 2D measurements.

Various conclusions can be drawn from this study regarding the practical conditions under which it is necessary to use the DWBA-based volume-integral scattering model with high-resolution 3-D digitizations of animal shape. Before discussing the range of practical conditions in further detail it should be noted that many of these conclusions are based on predictions of average quantities. Once averages are taken, either over a distribution of angles, lengths, shapes, or sizes, much of the structure seen in the individual pings will tend to be washed out. Consequently, the model predictions tend to agree better with each other.

As was mentioned earlier, little is known about the natural orientation distribution of elongated fluid-like zooplankton in their natural environment. Kils (1982), Sameoto (1980), and Endo (1993) have described tank measurements of the body orientations of Antarctic krill (Euphausia superba), and there have been similar studies (Miyashita et al., 
1996) performed on Euphausia pacifica. In a more recent study, Benfield et al. (2000) have found that the natural body orientation of Calanus finmarchicus, another member of the elongated fluid-like class of zooplankton, tends to be naturally oriented with the animal body axis vertically up, with a standard deviation of about $30^{\circ}$. Thus, based on the performance of the volume-integral model as compared to the lineintegral model, it can be concluded that if the natural distribution of orientations includes angles of incidence close to end-on, more accurate scattering predictions are possible with the volume-integral model. Likewise, there is little information currently available as to the variations in the material properties throughout the body volume (Foote, 1998; Yayanos et al., 1978). However, it is possible that the material properties will vary both along the lengthwise axis of the animals, as well as radially. Once quantified, it would be straightforward to include these volume inhomogeneities in the volume-integral model.

It is apparent from this investigation that the DWBAbased volume-integral scattering model, using highresolution 3D measurements of shape, reproduces the laboratory data for common shore shrimp under certain conditions. However, there are models that make use of simpler representations of animal shape (Stanton and Chu, 2000) that are also accurate over a narrow range of conditions. Whether or not it is necessary to make use of a sophisticated, but more complex, scattering model that incorporates highresolution measurements of animal shape may depend on the particular application. In fact, based on the results of the volume-integral model presented earlier, and depending on the specific applications, the largest sources of error in predicting scattering in realistic field situations may be dominated by the uncertainties in the material property and orientation distributions. Finally, if the animal proportions (i.e., the general shape and aspect ratio) scale with the animal size, it may be possible to create a database of scattering predictions from 3D measurements of a relatively small number of individuals.

\section{SUMMARY AND CONCLUSIONS}

In summary, an acoustic scattering model for fluid-like scatterers with complex shapes, which is based on the distorted wave Born approximation and incorporates highresolution three-dimensional digitizations of shape, has been developed. This model has a wide range of applicability, and has been applied in this study to the specific case of decapod shrimp (Palaeomonetes vulgaris), an elongated fluid-like scatterer, which closely resembles many members of the elongated fluid-like class of zooplankton. Detailed acoustic scattering measurements of live individual animals have been compared to the model predictions. These data, as well as the modeling results and predictions, have shown that the scattering process is highly complex and sensitive to the shape and size of the organisms, in addition to the angle of orientation, material properties, and acoustic frequency. This high level of variability indicates that sophisticated models are necessary to reproduce all aspects of the scattering process on a ping-by-ping basis.
High resolution CT scans have been used to accurately incorporate the full three-dimensional animal shape into the DWBA-based volume-integral scattering model. Comparisons have been made between the volume-integrated model and a DWBA-based line-integral scattering model that uses a 2D representation of animal shape. The model predictions agree reasonably well with each other, and with the ping-byping low-frequency narrow-band laboratory data, at angles close to broadside (Fig. 12). However, at angles close to end-on incidence, neither model successfully predicted TS value close to those observed in the ping-by-ping data, even at the lower frequencies (Fig. 12). It should be noted that CT scans were not available for the same animals from which the acoustic scattering data were collected; consequently, the volume-integral predictions were based on measurements of a scaled version of a different animal (scaled by aspect ratio). It has also been shown here that at higher frequencies the line-integral model predicts higher average scattering levels than those predicted by the volume-integral model or seen in the averaged data (Fig. 16), when averages are taken over all angles of orientation $\left(0-360^{\circ}\right)$ in one plane. The higher average scattering levels can be understood in terms of the creation of facets during the $2 \mathrm{D}$ digitization process. This was not the case with the volume-integral approach in which 3D CT scans were used to obtain the digitizations of animal shape. We conclude that care must be used with the lineintegral model, particularly at higher frequencies. In contrast, the DWBA-based volume-integral scattering model, which makes use of the high-resolution 3D measurements of animal shape, compared better with the averaged data (Fig. 16) over the full frequency range investigated.

One of the many aims of studies such as this one is to improve estimates of the numerical abundances of zooplankton as extracted from acoustic data obtained during field surveys, as well as to perform classification of zooplankton aggregations. However, accurately extracting this information from the acoustic field data remains a challenging problem. Investigations such as the one presented here, which combine a general scattering formulation with high-resolution morphological information and high-quality laboratory data, are key to the quantitative use of acoustics in studying zooplankton in the ocean.

\section{ACKNOWLEDGMENTS}

The authors would like to thank Darlene Ketten and Scott Cramer for performing the CT scans of the decapod shrimp, as well as to Hanu Singh and Ryan Eustice for help with the image analysis of CT images. This work was supported in part by the Woods Hole Oceanographic Institution Education Office. This is Woods Hole Oceanographic Institution Contribution Number 10425.

Anderson, V. C. (1950). "Sound scattering from a fluid-sphere," J. Acoust. Soc. Am. 22, 426-431.

Benfield, M. C., Davis, C. S., and Gallager, S. M. (2000). "Estimating the in situ orientation of Calanus finmarchicus on Georges Bank using the Video Plankton Recorder," Plankton Biol. Ecol. 47, 69-72.

Chu, D., Stanton, T. K., and Wiebe, P. H. (1992). "Frequency dependence of sound backscattering from live individual zooplankton,” ICES J. Mar. Sci. 49, 97-106. 
Chu, D., Foote, K. G., and Stanton, T. K. (1993). "Further analysis of target strength measurements of Antarctic krill at $38 \mathrm{kHz}$ and $120 \mathrm{kHz}$ : Comparison with deformed-cylinder model and inference of orientation distribution," J. Acoust. Soc. Am. 93, 2985-2988.

Chu, D., and Ye, Z. (1999). "A phase-conjugated distorted wave Born approximation representation of the bistatic scattering by weakly scattering objects: Application to zooplankton," J. Acoust. Soc. Am. 106, 17321743.

Chu, D., Wiebe, P. H., and Copley, N. (2000). "Inference of material properties of zooplankton from acoustic and resistivity measurements," ICES J. Mar. Sci. 57, 1128-1142.

Endo, Y. (1993). "Orientation of Antarctic krill in an aquarium," Nippon Suisan Gakkaishi 59, 465-468.

Everson, I. (1982). "Diurnal variations in mean volume backscattering strengths of an Antarctic krill (Euphausia superba) patch," J. Plankton Res. 4, 155-162.

Everson, I., Watkins, J. L., Bone, D. G., and Foote, K. G. (1990). "Implications of new acoustic target strengths for abundance estimates of Antarctic krill," Nature (London) 345, 338-340.

Foote, K. G., Everson, I., Watkins, J. L., and Bone, D. G. (1990). "Target strengths of Antarctic krill (Euphausia superba) at $38 \mathrm{kHz}$ and $120 \mathrm{kHz}$," J. Acoust. Soc. Am. 87, 16-24.

Foote, K. G., and Stanton, T. K. (2000). "Acoustical Methods," in ICES Zooplankton Methodology Manual, edited by R. Harris, P. Wiebe, J. Lenz, H. Skjoldal, and M. Huntley (Academic, London), Chap. 6, pp. 223-258.

Foote, K. G. (1990). "Speed of sound in Euphausia superba," J. Acoust. Soc. Am. 87, 1405-1408.

Foote, K. G. (1998). "Measurement of morphology and physical properties of zooplankton," in Digest of Colloquium on 'Recent advances in sonar applied to biological oceanography' (IEEE, Savoy Place, UK).

Greene, C. H., Stanton, T. K., Wiebe, P. H., and McClatchie, S. (1991). “Acoustic estimates of Antarctic krill," Nature (London) 349, 110.

Greenlaw, C. F. (1977). "Backscattering spectra of preserved zooplankton," J. Acoust. Soc. Am. 62, 44-52.

Hewitt, R. P. and Demer, D. A. (1991). "Krill abundance," Nature (London) 353, 310 .

Holliday, D. V., Pieper, R. E., and Kleppel, G. S. (1989). "Determination of zooplankton size and distribution with multi-frequency acoustic technology," J. Cons., Cons. Int. Explor. Mer 46, 52-61.

Holliday, D. V., and Pieper, R. E. (1995). "Bioacoustical oceanography at high frequencies," ICES J. Mar. Sci. 52, 279-296.

Kils, U. (1981) "The swimming behavior, swimming performance and energy balance of Antarctic krill, Euphausia superba," BIOMASS Scientific Series No. 3, 122.

Martin Traykovski, L. V., O’Driscoll, R. L., and McGehee, D. M. (1998). "Effect of orientation on broadband acoustic scattering of Antarctic krill Euphausia superba: Implications for inverting zooplankton spectral acoustic signatures for angle of orientation," J. Acoust. Soc. Am. 104, 21212135 .
McGehee, D. M., O’Driscoll, R. L., and Martin Traykovski, L. V. (1998). "Effect of orientation on acoustic scattering from Antarctic krill at 120 kHz," Deep-Sea Res., Part II 45, 1273-1294.

Medwin H., and Clay, S. C. (1998). Fundamentals of Acoustical Oceanography (Academic, Boston).

Miyashita, K., Aoki, I., and Inagaki, T. (1996). "Swimming behavior and target strength of isada krill (Euphausia pacifica)," ICES J. Mar. Sci. 53, 303-308.

Morse, P. M., and Ingard, K. U. (1968). Theoretical Acoustics (Princeton University, Princeton).

Sameoto, D. D. (1980). "Quantitative measurement of euphausiids using $120-\mathrm{kHz}$ sounder and their in situ orientation," Can. J. Fish. Aquat. Sci. 37, 693-702.

Stanton, T. K., Clay, C. S., and Chu, D. (1993a). "Ray representation of sound scattering by weakly scattering deformed fluid cylinders: Simple physics and application to zooplankton," J. Acoust. Soc. Am. 94, 34543462 .

Stanton, T. K., Chu, D., Wiebe, P. H., and Clay, C. S. (1993b). “Average echoes from randomly oriented random-length finite cylinders: Zooplankton models," J. Acoust. Soc. Am. 94, 3463-3472.

Stanton, T. K., Wiebe, P. H., Chu, D., Benfield, M. C., Scalon, L., Martin, L. V., and Eastwood, R. L. (1994). "On acoustic estimates of zooplankton biomass,” ICES J. Mar. Sci. 51, 505-512.

Stanton, T. K., Chu, D., and Wiebe, P H. (1996). "Sound scattering characteristics of several zooplankton groups,” ICES J. Mar. Sci. 53, 289-295.

Stanton, T. K., Chu, D., Wiebe, P. H., Martin, L. V., and Eastwood, R. L. (1998a). "Sound scattering by several zooplankton groups. I. Experimental determination of dominant scattering mechanisms," J. Acoust. Soc. Am. 103, 225-235.

Stanton, T. K., Chu, D., and Wiebe, P. H. (1998b). "Sound scattering by several zooplankton groups. II. Scattering models," J. Acoust. Soc. Am. 103, 236-253.

Stanton, T. K., Wiebe, P. H., and Chu, D., (1998c). "Differences between sound scattering by weakly scattering spheres and finite-length cylinders with applications to sound scattering by zooplankton," J. Acoust. Soc. Am. 103, 254-264.

Stanton, T. K., and Chu, D. (2000). "Review and recommendations for modeling of acoustic scattering by fluid-like elongated zooplankton: Euphausiids and copepods," ICES J. Mar. Sci. 57, 793-807.

Stanton, T. K., Chu, D., Wiebe, P. H., Eastwood, R. L., and Warren, J. D. (2000). "Acoustic scattering by benthic and planktonic shelled animals," J. Acoust. Soc. Am. 108, 535-550.

Urick, R. J. (1983). Principles of Underwater Sound (McGraw-Hill, New York).

Yayanos, A. A., Benson, A. A., and Nevenzal, J. C. (1978). "The pressurevolume-temperature (PVT) properties of a lipid mixture from a marine copepod, Calanus plumchrus; Implications for buoyancy and sound scattering," Deep-Sea Res. 25, 257-268. 